\title{
How to Convert One Green Photon Into Two Locomotives of Momentum
}

\author{
Terry B. Bollinger (1) 1 \\ ${ }^{1}$ Apabistia Research, Ashburn, VA, USA 20147
}

(Incepted 30 September 2021; Published 15 November 2021; Tweaked 2021-11-15.23:48)*

\begin{abstract}
The physics of photon momentum are straightforward mathematically but can produce surprisingly counterintuitive outcomes. A few simple calculations show how a single photon of green light can, in principle, impart two locomotive engines' worth of momentum without violating energy conservation. The calculation is one example of why quantum mechanics needs better accounting of linear momentum.
\end{abstract}

DOI: https://doi.org/10.48034/20210930

This is about: Photon momentum, energy conservation

\section{THE GOAL}

While extraordinarily valuable for keeping us alive in everyday situations, common-sense intuition is often problematic for extreme physics situations. The math often does not justify the shortcuts that our survivaloptimized brains try to take in such cases.

For example, everyone knows that momentum is just energy in kinetic form. We see this every day and know it from the equations. What is less apparent is how differently these two forms of energy behave when applied to ranges beyond everyday human experience. This paper describes one such case: Converting the energy of one photon of green light into momentum equivalent to two large locomotive engines running at top speed. No additional energy is involved in the conversion process, just that of the photon.

\section{CONVERTING PHOTON ENERGY INTO MOVING-OBJECT MOMENTUM}

The goal is to convert the energy of one zero-rest-mass photon into conventional momentum of moving masses.

Since energy is a scalar and momentum is a vector, this conversion requires converting the energy into a pair of opposing and thus mutually canceling momentum vectors - a classic Newtonian action-reaction pair. Figure 1 shows a straightforward method to accomplish this.

Published by Apabistia Press under the Creative Commons Attribution 4.0 International License. You are free to redistribute this article as you choose, provided only that you preserve the author attribution, article title, journal citation, and DOI.

${ }^{*}$ This document uses Kenobi versioning. The online name always points to the most recent document version, while obl, ob2,... suffixes indicate obsolete releases of the document.
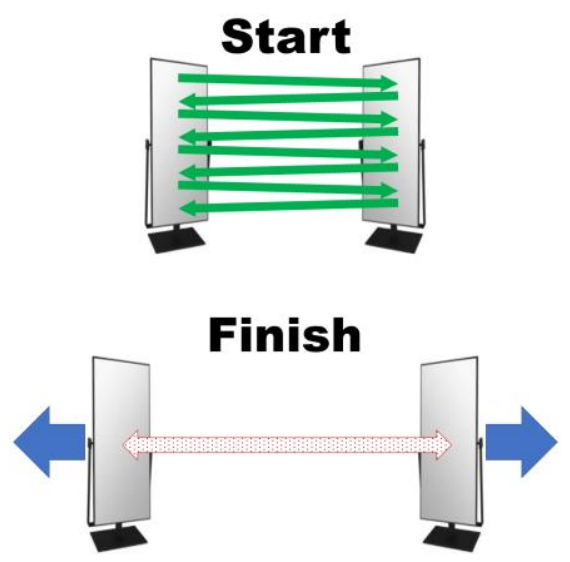

FIG. 1. Using reflectors to convert photons into momentum.

The green photon reflects coherently between the two mirrors, imparting a bit of momentum and energy to each time it reflects. Eventually, the energy of the zero-restmass photon is exhausted, at which point that energy has been converted fully into opposing momentum vectors in the two mirrors. The process seems straightforward, so much so that it can be modeled reasonably well by treating the photon as nothing more than a perfectly elastic ball. The main difference is that a ball slows down as it loses energy, while a green photon keeps light speed while losing energy by shifting its frequency down to red, infrared, and radio wavelengths. The conversion process thus does not appear to be particularly mysterious. Closer examination shows there are some surprises, however.

In everyday life, positioning two mirrors to face each other creates the same infinity-of-mirrors situation used in the figure. Depending on the quality of the mirrors, the images appear to go off into infinity. Real-life mirrors have losses that cause the images to dim. But by inspection alone, it is easy to see that once you factor out the dimming 
effects of imperfections in the mirrors, each new image pair looks exactly like the first one. The image brightness - the energy - is the same, the colors are the same, and the geometry is the same. What happened to the expected redshift? Also, what is happening in terms of momentum transfers? How quickly is photon momentum transferring into the mirrors?

One way to resolve this curious discrepancy is to assume that there is no momentum transfer. With this assumption, the mirrors remain in place, and there is no momentum transfer after all. In this situation, the photon would reflect forever, never losing energy.

Chaining the mirrors (Figure 2) creates this situation. The momentum imparted by each photon returned to it by a perfectly elastic rebound, just as would be the case for a perfectly elastic ball bouncing between plates.

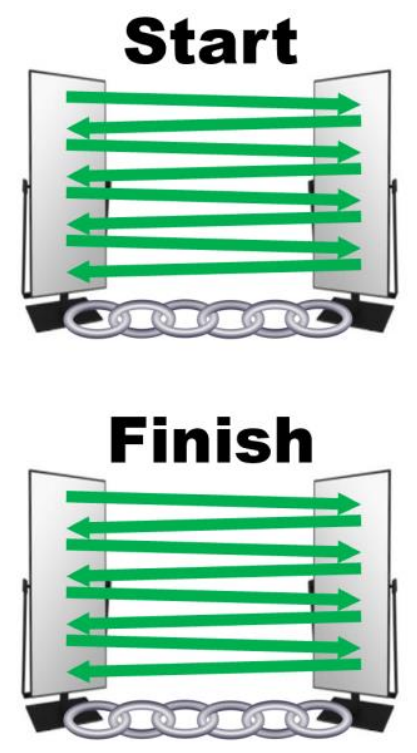

FIG. 2. Photon energy conservation with bound mirrors.

Alas, this answer is not very satisfying since it locks out momentum transfer entirely. Imagine instead two perfect mirrors floating in deep space, far away from gravity wells. In that situation, each mirror behaves like a specular (mirror-like) solar sail [1] [2]. As first theorized by the remarkable James Clerk Maxwell with his "molecular vortex" theory [3] that he later transformed into generic equations, light reflecting from an object should impart radiation pressure on that object. Lebedew [4] in the early 1900s and Nichols and Hull [5] [6] later all confirmed Maxwell's predictions. Macchi and Maragò [7] summarize historical and recent references to the theory and use of radiation pressure over diverse scales.

(It is worth pointing out that the better-known Crookes radiometer, also called a light mill, uses light only to power a jet-like reaction from heating diffuse gases [8] [9].
Light mills should not be confused with the entirely different phenomenon of radiative pressure.)

Thus after a century and a half of theory and more than a century of experimentation, including recent solar sail tests in space [10], it is clear that photons impart momentum each time they strike and reflect from a mirror. Quantizing radiation pressure requires photons that reflect perpendicularly from specular reflectors to impart twice their quantum momentum into the reflector [11], just as a physical ball would if bouncing perpendicularly and elastically between two plates in space. The radiation pressure theory first proposed by the remarkable Maxwell, well before (but leading into) the creation of his famous electromagnetic differential equations, remains the first and best prediction of this pressure, even after the emergence of quantum mechanics.

One notable feature of both photons and elastically bounding balls is this: How can they transfer twice their momentum per bounce? Why isn't momentum conserved?

\section{COSTS OF MOMENTUM PAIR CREATION}

Physics students learn very early that momentum, like energy, is always conserved. How can either a photon or a ball deposit twice its momentum when it bounces perpendicularly from a reflector? What is the source of the additional momentum? Momentum doubling is, at least at first glance, even more puzzling in the case of the photon since the energy of a photon is always $c$ times its momentum [12] [13]. How can a solar sail of a satellite visible from the earth receive twice the momentum of a photon without visibly impacting its color or energy?

The catch is that, unlike scalar energy, momentum is a vector quantity that can be created and destroyed in actionreaction pairs. As long as there is enough energy to create a new momentum pair, it is possible to create and add total momentum to isolated components of that system. (The system as a whole retains whatever momentum it had before the internal creation of new momentum pairs.)

More importantly - and directly related to why you do not see a red-shifted image of yourself when you look into a mirror - this momentum-pair creation process can be astonishingly energy-efficient. Photons reflected back to earth from orbiting satellites do lose a tiny bit of energy as they nudge the orbit of that satellite, solar-sail style. However, for a single photon striking any human-scale satellite, this photon energy lost is far too minute to be observed. Only in the quantum domain does this minute energy loss have the potential to impact an individual photon's behavior, and even then only in certain situations, as is discussed briefly at the end of this article.

Unintuitively, the most critical parameter for enabling the energy-efficient creation of momentum pairs is the mass of the mirrors. Specifically, the higher the mass of the mirrors, the more energy-efficient the process of 
creating momentum pairs becomes. Mirror mass is vital because momentum is the product of mass and velocity, so that high-mass objects can add momentum using meager velocity increases. The small velocity increases, in turn, permit the efficient creation of new momentum pairs.

Conversely, if the mirrors are low-mass, the elastic impact of a photon (or ball) accelerates the mirror much more. This acceleration of the mirror consumes more photon (or ball) energy of the photon, since for the mirror, $E_{k}=1 / 2 m v^{2}$. The higher consumption of photon energy is why an otherwise elastic reflection of a photon on a lowmass entity at atomic or particle scales is typically called a "scattering" event, one that significantly impacts both the photon and the object with which it interacts. Photons lose energy quickly in low-mass momentum transfer events and so can seldom create many momentum pairs. Such rapid energy dissipation is, for example, the fate of most photons emitted inside a dense gas or plasma.

Conversely, repeatedly reflecting photons (or balls) between two reflectors with arbitrarily higher masses can, in principle, create arbitrarily large numbers of momentum pairs. Thus, a single green light photon with a wavelength of $530 \mathrm{~nm}$, reflecting perfectly between two sufficiently massive reflectors can, in principle and with sufficient time, accumulate enough new photon-sized momentum pairs to add the equivalent of two large locomotive engines traveling at full speed in opposite directions and do so without the addition of any further energy.

\section{THE NUMBERS}

The numbers involved (see Appendix A) are daunting, however. For one green photon to reach the twolocomotive level of momentum creation, each of the reflectors must have a mass of $1.16 \times 10^{32} \mathrm{~kg}$ or 58.3 solar masses [14]. While the process deposits the equivalent of two photons of momentum with each reflection, the masses involved are so enormous that even if the reflectors are 1 meter apart - good luck on that bit of engineering - it takes 40 million times the universe's 13.77 billion years of history to accelerate the reflectors close to their final terminal velocity of 1.8 nanometers per billion years. Yet despite such absurd numbers, the scenario shows that, in principle, even a single green photon can generate locomotive-scale momentum pair sums with no further addition of energy. On a much smaller and more realizable scale, this same astonishing momentum-pair creation efficiency should enable future solar sails to travel between stars.

\section{THE QUANTUM PERSPECTIVE}

While the idea that one green photon can convert into two locomotives worth of momentum is an unexpected and amusing thought problem, it also illuminates a far more impactful issue: Linear momentum, in contrast to angular momentum (spin), is not quantized in quantum mechanics. This observation is, in one sense, obvious and even trivial since linear momentum, unlike angular momentum, does not occur in Planck units. However, the very existence of devices such as solar sails proves that in another sense, this lack of linear momentum quantization is anything but trivial, since it means the accumulation of indefinite quantities of energy from reflecting photons is both classically measurable and capable of altering the state of both the sail and the photons that impact it. It is easy to get into the habit of assuming that all behaviors in the quantum world are quantized. Linear momentum transfers in general, particularly the class of indefinitely high-energy-efficiency momentum transfers between lowmass quantum-scale objects and vastly larger classical objects, cannot safely be ignored. The wave function of a photon from the end of the dark ages may end up quantized in terms of energy and spin when observed in some radio telescope. However, the linear momentum of that same photon is never quantized, and so touches - transfers minuscule packets of momentum - with literally cosmic energy efficiency and scale to all the regions of the cosmos it has touched during its travels.

Both Schrödinger wave functions of fermions and the more puzzling photon wave functions [15] use an angular momentum framework. For example, the reason why fermionic, real-matter entities, especially smaller ones such as electrons, cannot stay located in one $x y z$ location forever is that their linear momentum components rotate in complex space at rates the differ by energy, causing the sum of such momentum waves to blur and diffuse over time. The reality of such momentum-wave expansion and recompression is particularly stark in recent work on single-atom heat engines [16], in which a single-atom wave function and its momentum components stand in with remarkable fidelity for entire multi-atom gases.

\section{WHEN 'NOT SEEING' IS JUST HIDDEN LOW-LEVEL MOMENTUM REMOVAL}

The observation that small quantum entities can lose vanishingly small but impactful quantities of linear momentum affects the interpretation of ghost imaging and path indistinguishability [17] where "unseen" photons provide imaging. While alternative paths do not witness the quantization of particles, they still remove momentum and so alter the energies of the final quantized photons. If this were not so, solar sails could not sail. Even when cosmically diluted, the impact of linear momentum cannot be ignored in quantum analysis [18].

\section{COLLAPSE AS MOMENTUM RESOLUTION}

Finally, there is this observation: The ease with which small objects distribute momentum makes them fragile in intriguingly ways. This is a topic for a future paper. 


\section{APPENDIX A: THE CALCULATION}

\section{A.1 RELEVANT CONSTANTS}

Photon momentum:

Photon energy:

$$
p=h / \lambda
$$

Planck's constant:

$$
E=h c / \lambda=p c
$$

$$
h=6.626 \times 10^{-34} \mathrm{~m}^{2} \mathrm{~kg} \cdot \mathrm{s}^{-1} \cong 6.63 \times 10^{-34} \mathrm{~J} \cdot \mathrm{s}
$$

Speed of light:

Green light:

$$
c=299792458 \mathrm{~m} / \mathrm{s} \cong 2.998 \times 10^{8} \mathrm{~m} / \mathrm{s}
$$

1 Joule:

$$
530 \mathrm{~nm}=530 \times 10^{-9} \mathrm{~m}=5.3 \times 10^{-7} \mathrm{~m}
$$

$\mathrm{J} / \mathrm{eV}$ :

$$
1 J=1 \mathrm{~kg}(\mathrm{~m} / \mathrm{s})^{2}
$$

$\mathrm{eV} / \mathrm{J}$ :

$$
1.602176634 \times 10^{-19} \mathrm{~J} / \mathrm{eV}
$$

$$
6.242 \times 10^{18} \mathrm{eV} / \mathrm{J}
$$

\section{A.2 MOMENTUM AND ENERGY OF ONE GREEN LIGHT PHOTON}

The momentum $p_{g}$ of one green-light photon with a wavelength $\lambda_{g}=5.3 \times 10^{-7} \mathrm{~m}$ is:

$$
\begin{aligned}
p_{g} & =h / \lambda_{g} \\
& =\left(6.63 \times 10^{-34} \mathrm{~m}^{2} \mathrm{~kg} \cdot \mathrm{s}^{-1}\right) /\left(5.3 \times 10^{-7} \mathrm{~m}\right) \\
& \cong 1.25 \times 10^{-27} \mathrm{~kg} \cdot \mathrm{m} / \mathrm{s}
\end{aligned}
$$

The energy $E_{g}$ of the same green-light photon is:

$$
\begin{aligned}
E_{g} & =p_{g} c \\
& =\left(1.25 \times 10^{-27} \mathrm{~kg} \cdot \mathrm{m} / \mathrm{s}\right)\left(2.998 \times 10^{8} \mathrm{~m} / \mathrm{s}\right) \\
& =3.7475 \times 10^{-19} \mathrm{~kg} \cdot(\mathrm{m} / \mathrm{s})^{2} \\
& =3.7475 \times 10^{-19} \mathrm{~J} \\
& =\left(3.7475 \times 10^{-19} \mathrm{~J}\right)\left(6.242 \times 10^{18} \mathrm{eV} / \mathrm{J}\right) \\
& =2.3391895 \mathrm{eV} \\
& \cong 2.34 \mathrm{eV}
\end{aligned}
$$

\section{A.3 MOMENTUM OF A LOCOMOTIVE ENGINE}

The question is how to replicate the momentum of a large locomotive engine running at maximum speed in each reflector. The mass $m_{L}$ of one particular locomotive, the GE AC6000CW diesel-electric is:

$$
m_{L}=196,000 \mathrm{~kg}=1.96 \times 10^{5} \mathrm{~kg}
$$

Its maximum speed is $75 \mathrm{mph} \cong 121 \mathrm{~km} / \mathrm{h}$ :

$$
v_{L}=1.21 \times 10^{5} \mathrm{~m} /(60 \cdot 60 \mathrm{~s}) \cong 33.6 \mathrm{~m} / \mathrm{s}
$$

The maximum-speed locomotive momentum $p_{L}$, which by definition is also the reflector momentum $p_{R}$, is:

$$
\begin{gathered}
p_{L}=p_{R}=m_{L} v_{L} \\
=\left(1.96 \times 10^{5} \mathrm{~kg}\right)(33.6 \mathrm{~m} / \mathrm{s}) \\
=6585600 \mathrm{~kg} \cdot(\mathrm{m} / \mathrm{s}) \\
\cong 6.59 \times 10^{6} \mathrm{~kg} \cdot(\mathrm{m} / \mathrm{s})
\end{gathered}
$$

The kinetic energy of each reflector is:

$$
E_{R}=1 / 2 m_{R} v_{R}^{2}
$$

Using one green photon as the energy source means each reflector gets half that energy:

$$
\begin{gathered}
E_{R}=1 / 2 E_{\lambda}=1 / 2 E_{g} \\
\Rightarrow 1 / 2 m_{R} v_{R}^{2}=1 / 2 E_{g} \\
\Rightarrow E_{g}=m_{R} v_{R}^{2}
\end{gathered}
$$

Solving for the reflector velocity gives:

$$
v_{R}=\sqrt{\frac{E_{g}}{m_{R}}}
$$

The momentum magnitude for one reflector (half the pair) is:

$$
p_{R}=p_{L}=m_{R} v_{R}=m_{R} \sqrt{E_{g} / m_{R}}=\sqrt{m_{R} E_{g}}
$$

Solving for $m_{R}$ :

$$
p_{R}=\sqrt{m_{R} E_{g}} \Rightarrow p_{R}^{2}=m_{R} E_{g} \Rightarrow m_{R}=\frac{p_{R}^{2}}{E_{g}}
$$

Putting in the numbers gives:

$$
\begin{array}{r}
m_{R}=\frac{\left(6.59 \times 10^{6} \mathrm{~kg} \cdot(\mathrm{m} / \mathrm{s})\right)^{2}}{3.7475 \times 10^{-19} \mathrm{~kg} \cdot(\mathrm{m} / \mathrm{s})^{2}} \\
=\frac{4.343 \times 10^{13} \mathrm{~kg}^{2} \cdot(\mathrm{m} / \mathrm{s})^{2}}{3.7475 \times 10^{-19} \mathrm{~kg} \cdot(\mathrm{m} / \mathrm{s})^{2}} \\
=1.16 \times 10^{32} \mathrm{~kg}
\end{array}
$$

Our sun's mass is $m_{S}=1.989 \times 10^{30} \mathrm{~kg}$. The standard symbol for one solar mass is $\mathrm{M} \odot$, the Unicode symbol for the sun. (It is also the ancient Chinese pictograph for the sun before scribes squared it into 日. Thus in terms of suns, each reflector needs a mass of:

$$
m_{R}=\frac{1.16 \times 10^{32} \mathrm{~kg}}{1.989 \times 10^{30} \mathrm{~kg}}=58.3 \mathrm{M} \odot
$$


The reflector momentum $p_{R}$ divided by the reflector mass gives the final velocity:

$$
\begin{aligned}
v_{R}=\frac{p_{R}}{m_{R}} & =\frac{6.59 \times 10^{6} \mathrm{~kg} \cdot(\mathrm{m} / \mathrm{s})}{1.16 \times 10^{32} \mathrm{~kg}} \\
= & 5.68 \times 10^{-26} \mathrm{~m} / \mathrm{s} \\
= & 5.68 \times 10^{-17} \mathrm{~nm} / \mathrm{s}
\end{aligned}
$$

One billion years (one giga-annum or eon) is roughly $1 G y=3.154 \times 10^{16} s$. Thus the velocity of one of the reflectors in nanometers per giga-annum $(\mathrm{nm} / \mathrm{Gy})$ is:

$$
v_{R}=1.8 \mathrm{~nm} / \mathrm{Gy}
$$

While that's a long wait to travel almost 2 nanometers, it pales in comparison to how long it takes for the oscillating photon to accelerate the reflectors up to full speed. Since the photon loses energy along the way, there is no finite limit to how long it takes for one green photon to convert all of its energy into reflector momentum. However, a reasonable least-bound for the time scale involved is to look at how long the acceleration process takes if one assumes the photon retains its original frequency until its final reflection.

Assuming the two $58.3 \mathrm{M} \odot$ reflectors are $1 \mathrm{~m}$ apart and good luck with that bit of engineering - then each reflection of the photon on a reflector amplifies and deposits twice its momentum $p_{g}$ on the reflector. Since an entire cycle between reflections is $2 m$, this works out as a simple deposition rate of $1 p_{g} / m$ of light travel. The distance $d_{R}$ in meters that the photon must travel at $c$ thus is:

$$
d_{R}=\frac{p_{R}}{p_{g}}=\frac{6.59 \times 10^{6}}{1.25 \times 10^{-27}}=5.272 \times 10^{33} \mathrm{~m}
$$

Converting to light-years $\left(9.461 \times 10^{15} \mathrm{~m} / \mathrm{ly}\right)$ gives:

$$
\begin{gathered}
d_{R}=5.57 \times 10^{17} l y \Rightarrow t_{R}=5.57 \times 10^{17} y r \\
=5.57 \times 10^{8} G y
\end{gathered}
$$

The universe is only 13.77 Gy old, so in units of alphaomega universe lifespans $T_{\alpha \omega}$ the time is:

$$
d_{R}=\frac{5.57 \times 10^{8} G y}{13.77 G y / T_{\alpha \omega}} \cong 40,450,000
$$

That is, it would take about 40 million times as long as the universe has existed for it to deposit enough newlycreated momentum pairs in the two $58.3 \mathrm{M} \odot$ to give them each the same momentum as a locomotive moving at full speed.
[1] L. Johnson, R. Young, E. Montgomery and D. Alhorn, "Status of Solar Sail Technology Within NASA," Advances in Space Research, vol. 48, p. 1687-1694, 2011.

[2] M. Leipold, M. Eiden, C. E. Garner, L. Herbeck, D. Kassing, T. Niederstadt, T. Krüger, G. Pagel, M. Rezazad, H. Rozemeijer and others, "Solar sail technology development and demonstration," Acta astronautica, vol. 52, p. 317-326, 2003.

[3] J. C. Maxwell, "III. On Physical Lines of Force," The London, Edinburgh, and Dublin Philosophical Magazine and Journal of Science, vol. 23, p. 12-24, 1862.

[4] P. Lebedew, "Untersuchungen über die Druckkräfte des Lichtes," Annalen der Physik, vol. 311, p. 433-458, 1901.

[5] T. Jensen, "Measuring the Pressure of Light: Pure Science at Dartmouth," Dartmouth Science History, 2002.

[6] E. L. Nichols, "Ernest Fox Nichols, 1869-1924: A Biographical Memoir," Biographical Memoirs of the National Academy of Sciences, 1929.

[7] A. Macchi and O. M. Maragò, "Light pressure across all scales: editorial," The European Physical Journal Plus, 26 May 2021.

[8] P. Gibbs, "How does a light-mill work," July 1996.

[9] O. Reynolds, "XVIII. On certain Dimensional Properties of Matter in the Gaseous State.," Philosophical Trans. of the Royal Society of London, p. 727-845, 1879.

[10] D. A. Spencer, B. Betts, J. M. Bellardo, A. Diaz, B. Plante and J. R. Mansell, "The LightSail 2 solar sailing technology demonstration," Advances in Space Research, vol. 67, p. 2878-2889, 2021.

[11] S. J. Ling, J. Sanny, B. Moebs, "16.5: Momentum and Radiation Pressure," Physics LibreTexts, 2021.

[12] A. Einstein, "On a Heuristic Point of View about the Creation and Conversion of Light," Ann. Physik, 17, 1905.

[13] Wikipedia, "Photon,” Wikipedia, 15 November 2021.

[14] T. Bollinger, "Efficient Momentum Pair Creation Using Massive Reflectors [to be published]," TAO Physics, vol. 2021, no. 10, 2021.

[15] C. T. Sebens, "Electromagnetism as Quantum Physics," Foundations of Physics, vol. 49, p. 365-389, 2019.

[16] J. Roßnagel, S. T. Dawkins, K. N. Tolazzi, O. Abah, E. Lutz, F. Schmidt-Kaler and K. Singer, "A single-atom heat engine," Science, vol. 352, p. 325-329, 2016.

[17] A. Hochrainer, M. Lahiri, M. Erhard, M. Krenn and A. Zeilinger, "Quantum Indistinguishability by Path Identity: The awakening of a sleeping beauty," arXiv preprint arXiv:2101.02431, 2021.

[18] T. Bollinger, "The Vacuum-Phonon (Sonon) Reinterpretation of QED," TAO Physics, vol. 2021, p. 0926, 26 September 2021. 